\title{
CONSTRUINDO INDICADORES PARA UMA AVALIAÇÃO AGRONÔMICA EM PARCELAS DE SISTEMAS AGROFLORESTAIS IMPLANTADAS EM ASSENTAMENTOS RURAIS, NO MUNICÍPIO DE ELDORADO DO CARAJÁS, PARÁ
}

\author{
Loyanne Lima Feitosa ${ }^{1}$; Luis Mauro Santos Silva ${ }^{2}$ \\ ${ }^{1}$ Discente do curso de Agronomia, Faculdade de Ciências Agrárias de Marabá (FCAM), Universidade Federal do \\ Pará, bolsista do PARD/PROPESP/UFPA, loy_lima@hotmail.com \\ ${ }^{2}$ Docente-pesquisador, UFPA/NCADR/LASAT, lmsilva@ufpa.br
}

\begin{abstract}
RESUMO: Recentemente o cenário de degradação na Amazônia demandou novas práticas e políticas governamentais de incentivo à recuperação dos agroecossistemas fortemente especializados com a pecuária. Dentre alguns dos sistemas de produção considerados alternativos, a implantação de Sistemas Agroflorestais (SAFs) surgiu como possibilidade concreta de diversificação e integração das atividades desenvolvidas pelas famílias assentadas na região. Este estudo objetivou construir um quadro inicial de indicadores para apoiar a avaliação agronômica de parcelas de SAFs, implantadas em Projetos de Assentamentos Eldorado e Moça Bonita no município de Eldorado do Carajás, estado do Pará. Através de uma ampla revisão de literatura foi possível elaborar o quadro de indicadores. Mas, a escassez de índices técnicos regionais, poucas referências teóricas regionais, ausência de estudos sobre avaliação de SAFs perante a realidade amazônica foram algumas das dificuldades encontradas. Para uma avaliação mais detalhada das parcelas de SAFs, ainda é necessário ponderar os indicadores levantados para assim se apoderar de uma reflexão.
\end{abstract}

PALAVRAS-CHAVE: Indicador agronômico, sistemas agroflorestais, assentamento rural.

\section{CONSTRUCCIÓN DE INDICADORES PARA UNA EVALUACIÓN AGRONÓMICA EN PARCELAS DE SISTEMAS AGROFORESTALES ESTABLECIDOS EN ASENTAMIENTOS RURALES, EN EL MUNICIPIO DE LDORADO DO CARAJÁS, PARA}

RESUMEN: Recientemente, la etapa de la degradación de la Amazonía llamado para nuevas prácticas y políticas gubernamentales para fomentar la recuperación de los agroecosistemas altamente especializado con el ganado. Entre algunos de los sistemas de producción alternativos considerados, el establecimiento de Sistemas Agroforestales (SAF) se ha convertido en una posibilidad real de la diversificación y la integración de las actividades de las familias asentadas en la región. Este estudio describe un marco inicial de indicadores para apoyar la evaluación agronómica de las parcelas de los SAF implementados en los proyectos de asentamiento Eldorado e Moça Bonita en el municipio de Eldorado do Carajás, estado de Pará. A través de una extensa revisión bibliográfica fue posible elaborar el indicador de la mesa. Pero la escasez de los índices técnicos, regionales referentes teóricos pocos, la ausencia de estudios de evaluación del SAF a la realidad del Amazonas fueron las dificultades encontradas. Para una evaluación más detallada de las porciones de SAF, todavía es necesario ponderar los indicadores de lo que elevó a apoderarse de una reflexión.

PALABRAS CLAVE: Indicador agronómico, agroforestal, asentamiento rural. 


\section{INTRODUÇÃO}

Na década de 1960, o governo militar implementou uma política desenvolvimentista, como conseqüências dessa política houve um intenso fluxo migratório para as regiões de fronteiras agrárias entre as décadas de 1970 e 1980, promovendo um palco de conflitos fundiários e exploração indiscriminada dos recursos naturais na região (TOURRAND; VEIGA, 2003).

Nessa lógica de exploração a agricultura familiar manteve o predomínio do sistema de corte-queima, onde áreas de florestas nativas foram substituídas progressivamente por áreas de monocultivo de pastagens, consolidando uma lógica hegemônica de pecuária extensiva como a principal atividade produtiva nos sistemas de produção desta região (VEIGA et al., 2007). Progressivamente o cenário amazônico passou a apresentar pastagens em diferentes estágios de degradação assim a especialização da pecuária extensiva tornouse inviável para a sustentabilidade dos agroecossistemas amazônicos (SILVA, 2009).

Mais recentemente, este cenário de degradação demandou novas práticas e políticas governamentais de incentivo à recuperação dos agroecossistemas fortemente especializados com a pecuária. Dentre alguns dos sistemas de produção considerados alternativos, a implantação de Sistemas Agroflorestais (SAFs) surgiu como possibilidade concreta de diversificação e integração das atividades desenvolvidas pelas famílias assentadas na região (SILVA, 2009). Em alguns estudos já é possível notar que a adoção de SAFs se consolida como opção na recuperação de áreas impactadas ecologicamente, devido á recorrente derrubada de florestas para a formação de pastagens, além de proporcionar a diversificação das atividades produtivas (COSTA et al., 2006).

Levando em consideração que os SAFs se apresentam como uma alternativa de diversificação dos sistemas produtivos realizados pela agricultura familiar e possibilitam agroecossistemas com bases amplas de sustentabilidade (ambiental, social e econômica). Este estudo objetivou construir um quadro inicial de indicadores para apoiar a avaliação agronômica de parcelas de SAFs, implantadas em Projetos de Assentamentos Eldorado e Moça Bonita no município de Eldorado do Carajás, estado do Pará.

\section{METODOLOGIA}

A primeira etapa do projeto de pesquisa intitulado "Adaptando ferramentas multidimensionais para avaliar a qualidade de vida das famílias assentadas no território sudeste do Pará." possibilitou que Nogueira (2010) caracterizasse e avaliasse a sustentabilidade destes agroecossistemas

\footnotetext{
${ }^{1}$ Esta Cooperativa de Prestação de Serviços Técnicos foi definida como prioritária para o estudo por estar envolvida no processo de acessória técnica aos assentamentos que implantaram SAFs no município de Eldorado do Carajás.
} 
utilizando um quadro de indicadores, já anteriormente validado por Silva (2008). Este estudo faz parte da segunda etapa do referido projeto, esta em andamento em fase de sistematização e análise de dados. Vem sendo realizado nos assentamentos Eldorado e Moça Bonita, ambos localizados na região do sudeste paraense, no município de Eldorado do Carajás, Pará. Estes projetos de assentamentos foram selecionados por apresentarem características peculiares como presença de SAFs implantados via Pronaf Floresta $^{2}$.

O Projeto de Assentamento Eldorado (PA), está localizado cerca de $20 \mathrm{~km}$ de distancia da sede municipal. Foi criado no ano de 1986, em meio a um conturbado processo de desapropriação, foram assentadas 135 famílias. Existem apenas $20 \%$ da área com cobertura vegetal nativa (COOPSERVIÇOS, 2004). Os solos pertencem as classes III e IV (de acordo com a classificação de solos), Segundo Lima (2005) são caracterizados pelo acentuado declive, podem sofrer erosão e são cultiváveis mediante práticas de conservação.

O PA Moça Bonita foi ocupado no ano de 1997, mas somente em 1999 foi decretada sua criação. Conta com uma área total de 3.415 há, assentando aproximadamente 75 famílias. Está localizado a $30 \mathrm{~km}$ de distancia da sede do

\footnotetext{
${ }^{2}$ A linha de crédito Pronaf Floresta lançada em 24 de junho de 2002 através da resolução $\mathrm{n}^{\text {o }} 3.001$ visa à expansão, conservação e manejo da cobertura florestal dos biomas brasileiros, pela implementação de projetos recuperação de áreas degradadas, de silvicultura e de sistemas agroflorestais (GONÇALVES et. al., 2009).
}

município de Eldorado dos Carajás. Compreende solos com capacidade de uso nas classes II, III e IV, apresentam terras boas para cultivos, com declividade, limitações quanto a baixa fertilidade. Apresenta $66 \%$ de cobertura vegetal nativa (COOPSERVIÇOS, 2001).

Este estudo limitou-se a avaliar apenas três parcelas de SAFs (a primeira no PA Eldorado e as outras duas no PA Moça Bonita). Através de consultas em referenciais teóricos procurou-se construir um quadro de indicadores agronômicos adaptáveis a avaliar parcelas de SAFs no contexto Amazônico.

Com base no referido quadro alguns passos metodológicos da pesquisa de campo já puderam ser realizados como: aplicação do questionário semi-estruturado, registros fotográficos, observações in locu; obtenção do Relato de experiência do Técnico responsável por acompanhar a implementação do projeto Pronaf Floresta, através de registros em manuscrito e acesso ao acervo documental da COPSERVIÇOS; coleta de amostras de solo fresco por meio de tradagem; obtenção do Diâmetro Altura do Peito (DAP) das espécies florestais e contagem do número indivíduos, espécies exóticas e nativas nas três parcelas de SAFs.

\section{RESULTADOS E DISCUSSÃO}

Os SAFs foram implantados via Pronaf Floresta no ano de 2006, em ares de áreas aproximadamente de 1 hectar, na terceira parcela predominava o pasto como 
antecendente vegetal e nas outras duas capoeira. O modelo de SAFs implantado era composto basicamente pela seguinte diversidade de espécie, Eucalipto (Eucalyptus spp.), Mogno (Swietenia macrophylla King.), Teca (Tectona grandis L.f.), Castanha-doPará (Bertholletia excelsa H.B.K), Paricá (Schizolobium amazonicum), Açaí (Euterpe olerace Mart) e Cupu-açu (Theobroma grandiflorum). Em uma análise preliminar Nogueira (2010) observou que a implantação dos SAFs nessas áreas promoveu a recuperação de parte da cobertura vegetal arbórea perdida com a introdução do pasto.

$\mathrm{Na}$ tentativa de aprofundar a análise iniciada por Nogueira (2010) e realizar uma avaliação agronômica de caráter mais complexo, analisando as relações existentes no subsistema SAF - Família. Buscou-se construir um quadro de indicadores agronômicos adaptáveis para avaliar parcelas de sistemas agroflorestais no contexto amazônico. A escassez de índices técnicos regionais, poucas referências teóricas regionais, ausência de estudos sobre avaliação de SAFS perante a realidade amazônica foram algumas das dificuldades encontradas durante a elaboração do quadro de indicadores. Pouco se tem avançado em reflexões sobre a consolidação de indicadores agronômicos, inibindo uma avaliação mais precisa dos impactos positivos e negativos em relação à sustentabilidade (ambiental, social e econômica, por exemplo) dos SAFs na Amazônia. 
QUADRO 01: Síntese geral dos indicadores de avaliação agronômica adaptáveis ao contexto amazônico

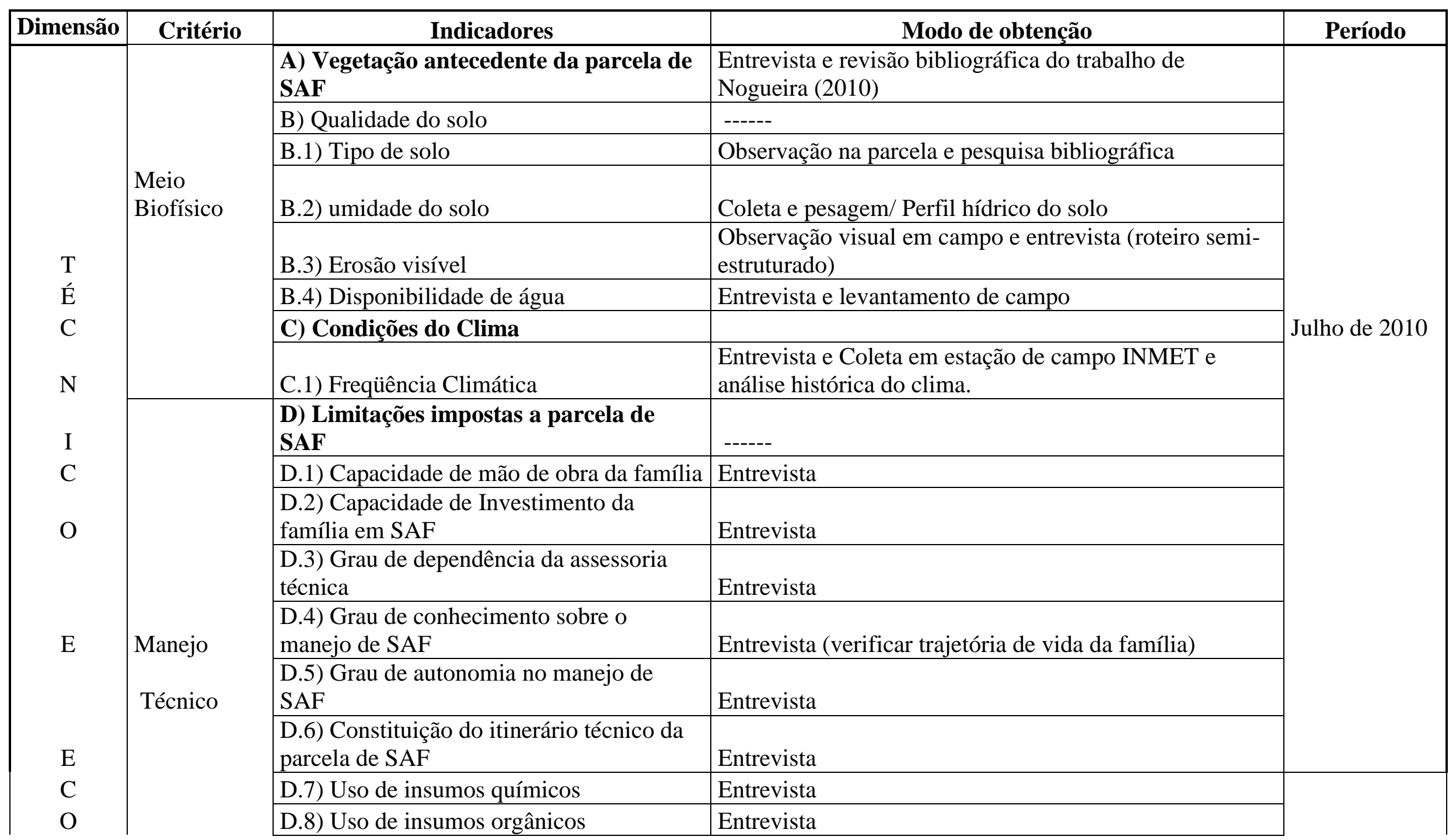




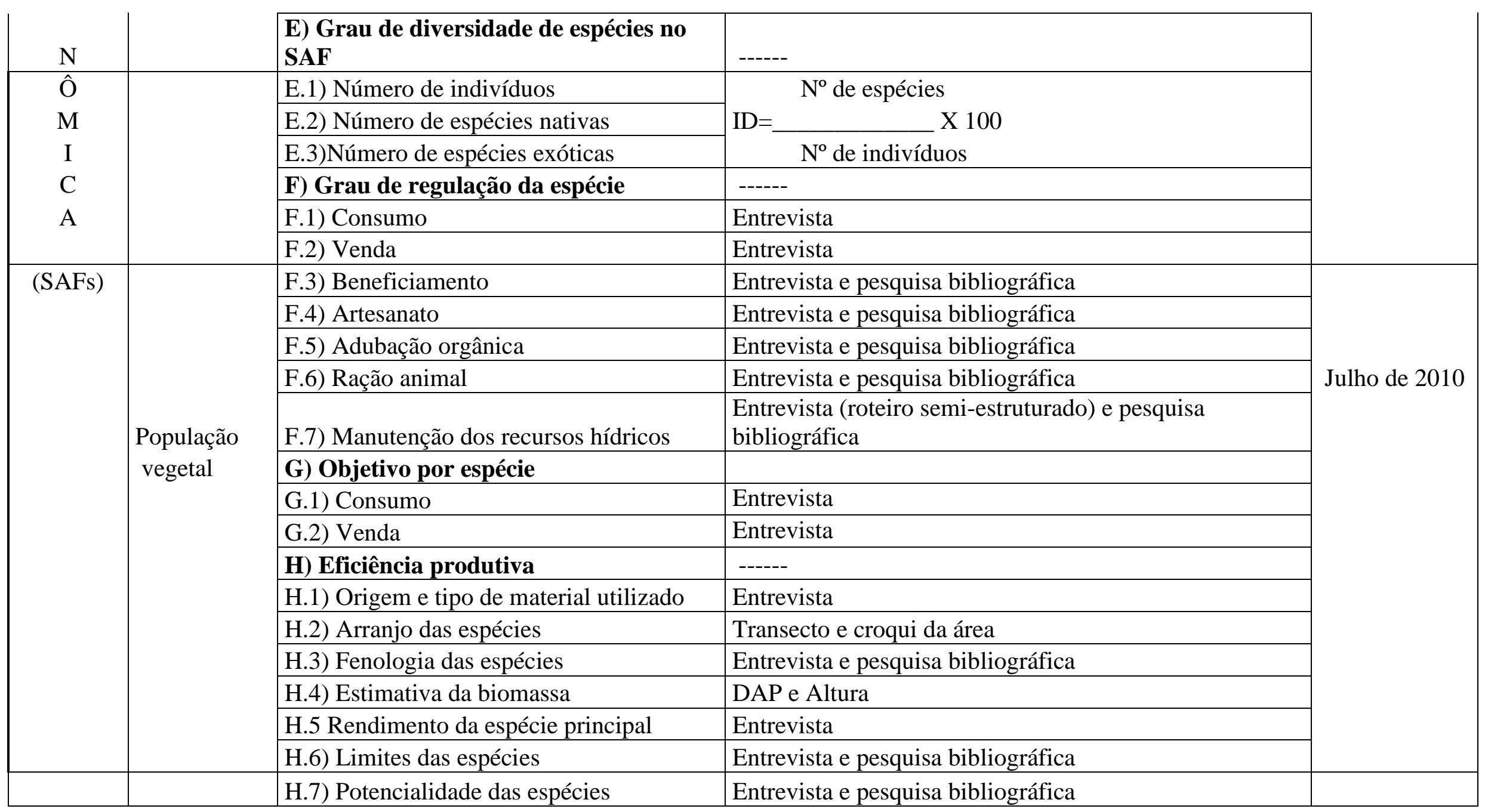




\section{CONCLUSÕES}

A dificuldade de compor o quadro de indicadores de avaliação de SAFs se explica pela escassez de pesquisas sobre referenciais técnicos regionais. A necessidade de ampliação deste debate e de pesquisas nesse âmbito eleva este trabalho (que encontrasse em processo de andamento) ao caráter inovador, o que de certa forma contribui e impulsiona a academia em um processo de continuidade desta construção junto aos atores locais. Com relação à avaliação propriamente dita, ainda é necessário ponderar os indicadores levantados para assim se apoderar de uma reflexão que permita uma avaliação mais detalhada das parcelas de SAFs.

\section{REFERÊNCIAS}

COSTA, R. B.; ARRUDA, E. J.; OLIVEIRA, L. C. S; PAULINO, V. T. Sistemas agrossilvipastoris como alternativa sustentável para a agricultura familiar. Revista Internacional de Desenvolvimento Local. Vol. 3, N. 5, Set. 2006.

GONÇALVES, K. G.; COUTINHO, M. P.; CASTELLANI \& PEDREIRA, F. L. de O. O acesso dos agricultores familiares ao crédito pronaf florestal e à assistência técnica e extensão rural em atividades florestais. In: CONGRESSO BRASILEIRO DE SISTEMAS AGROFLORESTAIS, VII. Anais... Brasília, 2009. CD-ROM. 4 p.

LIMA, M. R. de C. Avaliação de Propriedades Rurais: Manual básico. $2^{\circ}$ edição revistada e atualizada. São Paulo, 2005, 27p

NOGUEIRA, A. C. N. Sustentabilidade de Agroecossistemas Familiares que implantaram no município de Eldorado do Carajás, Pará. Trabalho de Conclusão de
Curso (TCC)- Faculdade de Agronomia, Universidade Federal do Pará - UFPA, 2010. 86p.

$\begin{array}{llll}\text { PLANO DE } & \text { DESENVOLVIMENTO DO } \\ \text { PROJETO } & \text { DE } & \text { ASSENTAMENTO } \\ \text { ELDORADO. Marabá: } & \text { INCRA } & \text { (SR27), } \\ \text { Copserviços, 2004.91p } & & \end{array}$

PLANO DE DESENVOLVIMENTO SUSTENTÁVEL DO PROJETO DE ASSENTAMENTO MOÇA BONITA. Marabá: INCRA(SR27); Copserviços,2001, 92p.

SILVA, L. M. S. Adaptando ferramentas multidimensionais para avaliar a qualidade de vida das famílias assentadas no território sudeste do Pará. Plano de Atividades, UFPA/PROPESP/PARD, 2009, $3 p$.

TORRAND, J. F.; VEIGA, J. B. Viabilidade de Sistemas Agropecuários na Agricultura Familiar da Amazônia. Embrapa Amazônia Oriental, Belém, 2003.

VEIGA, I.; OLIVEIRA, M. C. De O. \& BENTES, F. Políticas públicas e dinâmicas locais. UFRGS, RS, 2007, 109-135 p.

\section{AGRADECIMENTOS}

O presente trabalho foi realizado com o apoio do Programa Auxílio ao Recém-Doutor (PARD/PROPESP/UFPA). 